\title{
ETAPAS DEL CAMBIO CONDUCTUAL ANTE \\ LA INGESTA DE FRUTAS Y VERDURAS, CONTROL DE PESO \\ Y EJERCICIO FÍSICO DE ESTUDIANTES DE LA UNIVERSIDAD \\ DEL DESARROLLO, SEDE CONCEPCIÓN, CHILE
}

\author{
STAGES OF BEHAVIORAL CHANGES IN RELATION \\ TO INTAKE OF FRUITS AND VEGETABLES, WEIGHT CONTROL \\ AND PHYSICAL EXERCISE OF STUDENTS IN THE UNIVERSITY \\ OF DESARROLLO, CONCEPCION, CHILE
}

\author{
Miguel Ángel López E. \\ Escuela de Nutrición y Dietética, Facultad de Odontología, \\ Universidad del Desarrollo, Concepción, Chile
}

\begin{abstract}
Background: The study of behavior associated with the consumption of fruit and vegetables, weight control and physical activity in young adults following the Transtheoretical Model of Prochaska is a crucial aspect when assessing the impact that these three dimensions have as preventive risk factors for diseases linked to circulatory system and cancer. Objective: To analyze stages of behavioral changes concerning the consumption of fruit and vegetables, weight control and physical activity in students according to gender and nutritional status. Design: Analytical cross study with a stratified design of 184 students of both sexes between 18 and 28 years of age. Results: Women with normal nutritional status were significantly associated with the stage "preparation to action" (13,59\%) and "action" $(10,87 \%)$ with respect to women in weight control. Men with good nutritional status were associated with periods of "maintenance" to exercise (15,22\% of 184 students). In women, 59,6\% and 46.3\% of men were in preparation for action with respect to fruit and vegetable consumption $(p<0,05)$. Conclusion: Men and women perceive that only a normal nutritional status is a guarantee of good health, since boys do not give importance for the long-term weight control, and girls did not give any relevance to exercise in the long run.

Key words: Transtheoretical Model, fruit and vegetables intake, weight control, exercise, gender.
\end{abstract}

Este trabajo fue recibido el 11 de Enero de 2008 y aceptado para ser publicado el 12 de Agosto de 2008.

\section{INTRODUCCIÓN}

La carga de las enfermedades cardiovasculares, cáncer, diabetes, obesidad y otras enfermedades crónicas está aumentando rápidamente en todo el mundo. En 2001, las enfermedades crónicas fueron responsables de aproximadamente el $59 \%$ de los 56,5 millones de defunciones totales registradas en el mundo y el $46 \%$ de la carga mundial de morbilidad (1). En Chile, también existe un alto porcentaje atribuido a ellas, donde su valoración medida a través del riesgo cardiovascular es la pieza clave en la valoración integral de dicho sujeto ya que determina actitudes terapéuticas de acuerdo a dicho riesgo (2).
Más y más se desarrolla conciencia que los riesgos para la salud no solamente se deben a las condiciones ambientales, ya que éstas pueden ser alteradas por el propio hombre al satisfacer necesidades y afrontar los desafíos en el curso de su vida (3), en relación al peso corporal, a pesar de que diversos estudios de heredabilidad se ha sugerido que aproximadamente entre el $40 \%$ y el $70 \%$ de la variabilidad en el peso puede atribuirse a factores genéticos.

El incremento en la prevalencia de la obesidad que se ha observado en las últimas décadas en la mayoría de los países desarrollados, no se explica por mecanismos genéticos (4), en donde la ingesta calórica asociada a 
un consumo exagerado de grasas en la población adulta entre 25 y 64 años (5), lo que en conjunto desencadenan problemas de malnutrición por exceso, que a su vez se incrementa por el sedentarismo. Esto se refleja en un estudio en adultos laboralmente activos, con actividad de oficina en la Región Metropolitana, de los cuales el $13 \%$ de los que poseen estudios universitarios sufren obesidad, cifra significativamente inferior si se compara al 33\% de trabajadores con estudios básicos o medios incompletos.

Respecto al sedentarismo este mismo estudio refleja que el $82,5 \%$ de los adultos con actividad laboral de oficina y estudios universitarios se declara sedentario, cifra que no es diferente al compararlo con trabajadores con instrucción técnica completa, media completa y básica o media incompleta (6).

Los resultados de la Encuesta Nacional de Calidad de Vida y Salud 2000 (ECVS), indicaron que el 47\% de la población chilena dice consumir verduras y frutas todos los días (7). La versión 2006 de la ECVS señaló que el 47 y el $53 \%$ consumían frutas y verduras todos los días, respectivamente (8).

El nuevo informe de la Organización de Naciones Unidas para la Agricultura y Alimentación (del inglés, FAO) y la Organización Mundial de la Salud (OMS) recomienda realizar actividad física de moderada intensidad durante al menos una hora al día, aumentar el consumo de frutas y hortalizas frescas; además del recorte de la cantidad de sal en la dieta, reducir la energía y alimentos ricos en grasas saturadas y azúcares añadidos, tales como las que se encuentran en aperitivos, alimentos procesados y en bebidas (1). La medición de estas variables pasa además por una actitud del individuo, donde la capacidad de cambio de conductas no deseadas implica diferencia entre el éxito o el fracaso del empoderamiento efectivo de conductas saludables.

En la actualidad se enfatiza la necesidad de utilizar diversas teorías y modelos educativos cuando se diseñan y realizan intervenciones orientadas a lograr cambios de conducta que contribuyan a prevenir y controlar la obesidad y otras enfermedades crónicas. Todos ellos plantean que es esencial explorar el contenido actual de las creencias, percepciones, expectativas y valores en cada grupo, utilizando métodos cuantitativos y cualitativos como encuestas, entrevistas en profundidad o grupos focales, que tienen la ventaja de entregar valiosa información sobre aspectos subjetivos que explican el comportamiento de las personas (9). Sin esta debida información, no será posible diagnosticar la real relevancia del problema ni menos generar proyecciones de sus posibles nexos ni consecuencias en el estado de salud física a futuro, dado que la principal característica es que son un grupo de enfermedades y/o trastornos degenerativos que no causan síntomas aparentes al individuo.

El Modelo Transteórico (MT) del cambio del comportamiento en salud, llevado a cabo en 1979 por James Prochaska, se consolidó durante los años noventa como una de las propuestas más innovadoras en el área de promoción de la salud y prevención de la enfermedad (10). Ella ofrece posibilidades para planear y ejecutar intervenciones a partir de las características específicas de las poblaciones a quienes están dirigidas las acciones, quien detecta cinco etapas definidas: precontemplación, contemplación, preparación, acción y mantenimiento. Diversas publicaciones reconocieron la capacidad del modelo para describir y explicar las distintas etapas que son comunes a la mayoría de procesos de cambio comportamental y que a principio de los noventa fue progresivamente incorporado en las investigaciones e intervenciones de un amplio número de comportamientos ya reconocidos como de riesgo para la salud (10).

La etapa de precontemplanción se caracteriza porque la persona no ha considerado seriamente la idea de cambiar la conducta, la contemplación es una etapa de ambivalencia, es decir, por una parte el sujeto considera la necesidad de dejar su patrón de conducta, pero por otra no se encuentra en condiciones de hacerlo antes de seis meses; luego, viene la etapa de preparación a la acción, donde se adopta un planteamiento serio de cambio, generalmente asociado a la aparición de síntomas o experiencias cercanas de enfermedad; para evolucionar a la etapa de acción, en la cual el sujeto ha realizado los pasos necesarios para cambiar, y terminar con la etapa de mantenimiento de la acción, donde la persona sostiene por más de seis meses una conducta determinada evitando las recaídas para lo cual ha introducido cambios en su estilo de vida o muerte (11).

Recientemente, el MT se usa en el diseño de intervenciones facilitadoras de comportamientos saludables como el consumo de una dieta adecuada (12-14) y la adopción de prácticas deportivas regulares (15-17). Considerando el análisis de los hábitos alimentarios en la ingesta de fibra dietética, control de peso y actividad física se lograrán diseñar intervenciones específicas en función a la etapa conductual específica que se encuentre el individuo y evaluar el progreso de éste (18).

Considerando el perfil epidemiológico y nutricional actual del país, las prioridades deben continuar orientándose hacia la promoción de un estilo de vida (EV) más saludable, con especial énfasis en la alimentación y la actividad física, así como en la prevención de la obesidad y las enfermedades crónicas asociadas (19).

Este trabajo tiene por objetivo conocer y estudiar las etapas de cambio conductual referente a la ingesta de 
frutas y verduras, control de peso y ejercicio físico según estado nutricional y género de estudiantes universitarios. De los resultados obtenidos dependerán futuros diseños de modelos educativos que contribuyan a modificar hábitos respecto a estas tres dimensiones o mantener las conductas saludables en ellos.

\section{SUJETOS Y MÉTODO}

La investigación presenta un diseño analítico de corte transversal, la cual se desarrolló entre el 1 al 31 de octubre de 2007. De una población de 2810 estudiantes de pregrado registrados hasta el 1 de julio de 2007, se calculó el tamaño muestral con un nivel de confianza al $95 \%$, error muestral del $7 \%$ y una proporción teórica del $50 \%(\mathrm{p}=0,5)$, arrojando una muestra de 184 alumnos. Se diseñó un muestreo a las 18 carreras de la Universidad del Desarrollo sede Concepción, donde el primer paso fue seleccionar uno de los cursos de cada carrera de forma aleatoria, y luego al sujeto de estudio al azar sistemático según el listado de curso. Se excluyeron aquellas estudiantes que al momento de la investigación se encontrasen en período de gestación, condición que obliga a la mantención de un régimen dietético específico según sea el período que se encuentre. A los estudiantes seleccionados se les manifestó la intención y objetivo de la investigación y las mediciones a realizar, dejando constancia de su aceptación y participación a través de un protocolo de consentimiento informado y declaración voluntaria, luego de la aprobación del Comité de Ética de la Facultad. Las mediciones antropométricas y la aplicación de la encuesta para determinar las etapas del cambio en que se encuentran los encuestados, fueron realizadas por alumnos previamente entrenados de la Carrera de Nutrición y Dietética.

Se aplicó un cuestionario elaborado y validado por el Instituto de Nutrición y Tecnología de los Alimentos (INTA) de la Universidad de Chile (20), el cual mide etapas de la conducta en tres dimensiones (cuatro preguntas adaptadas del Instituto Nacional del Cáncer de Estados Unidos para determinar la etapa del cambio en el consumo en ingesta de frutas y verduras, una pregunta que determina la etapa del sujeto frente al ejercicio físico, y cuatro preguntas para establecer la conducta frente al control de peso). Este instrumento tiene una duración estimada de 10 minutos. Además del cuestionario, se incluyó la medición de peso con balanza digital marca SECA 881 digital con precisión de $0,01 \mathrm{~kg}$ y talla con un altímetro marca SECA modelo 225, con precisión es de $1 \mathrm{~mm}$, con el objeto de determinar el estado nutricional según el índice de Quetelet para adultos (21).

La información se analizó utilizando la prueba de Chi2 si las frecuencias esperadas son mayores a cin- co. Si lo anterior no fuera cumplido, se recurrió a los coeficientes V de Cramer y Gamma, según variables nominales u ordinales, respectivamente. La comparación de porcentajes de hombres y mujeres según sean los cambios conductuales fueron analizados según la prueba z para diferencias de proporciones. En el caso de la comparación de medias en dos grupos se usaron las pruebas de t-Student o Mann-Whitney, según varianzas homogéneas o heterogéneas, respectivamente. Se usó un nivel de significación menor a 0,05. La información fue analizada con el programa SPSS 15,0.

\section{RESULTADOS}

Se estudió a 104 mujeres $(56,52 \%)$ y 80 varones $(43,48 \%)$, de 18 a 28 años de edad (20,41 + 1,92 años). En términos generales, el peso promedio del grupo estudiado fue de $65,73+12,85$ kilos, con 1,66 + 0,09 $\mathrm{m}$ de estatura, arrojando un índice de masa corporal (IMC) de 23,74 + 3,38 kg/mt2. El 2,17\% de la muestra se diagnosticó como enflaquecido $(\mathrm{n}=4)$, el $68,48 \%$ presentó un estado nutricional normal $(\mathrm{n}=126)$, aquellos con sobrepeso son el $22,83 \%$ del grupo estudiado $(n=42)$ y los considerados con obesidad representan al 6,52\% de ellos $(n=12)$. La tabla 1 muestra valores medios de las características de los estudiantes investigados según género, observándose diferencias significativas de los parámetros antropométricos.

En cuanto al cambio conductual en relación al consumo de frutas y verduras, la tabla 2 muestra que no existe evidencia suficiente para afirmar que las etapas del cambio conductual de los estudiantes está asociado a la ingesta de frutas y verduras $(\mathrm{P}=0,228)$. Sin embargo, el sexo se asoció significativamente respecto a las dimensiones de control de peso y ejercicio físico, respectivamente $(\mathrm{P}<0,001)$.

Respecto a la ingesta de frutas y verduras, la tabla 2 indica que las mujeres presentaron significativamente mayor porcentaje en cuanto a la preparación a la acción respecto a los varones $(\mathrm{p}<0,05)$. Cabe señalar que no se observó a ninguna persona que se encuentre en la etapa de mantenimiento a la acción de ingesta de frutas y verduras y ninguno de los varones estaba en una etapa de preparación a la acción, mientras que en mujeres se observó sólo el $1 \%$.

Al estudiar la dimensión control de peso, son más varones que damas los que se encuentran en la etapa de precontemplación, mientras que las mujeres presentaron conductas de preparación a la acción y acción significativamente mayor respecto a los varones $(\mathrm{p}<0,001 \mathrm{y}$ $\mathrm{p}<0,01$, respectivamente). Por último, para el ejercicio físico se observó que las etapas de contemplación, preparación a la acción y la acción se encuentra concentrada 
significativamente en mujeres respecto a los hombres $(\mathrm{p}<0,001, \mathrm{p}<0,001$ y $\mathrm{p}<0,05$, respectivamente). Caso contrario ocurre con la conducta de mantenimiento de la acción del ejercicio físico, destacando que son los hombres quienes porcentualmente presentan más este rasgo que las mujeres ( $\mathrm{p}<0,001)$.

Al estudiar la posible asociación del estado nutricional y género en relación a las etapas del cambio conductual en función a la ingesta de frutas y verduras de los estudiantes investigados, la tabla 3 muestra que el $36,41 \%$ de los estudiantes no presentó interés en cambiar la conducta respecto al consumo de frutas y verduras, mientras que el 53,8\% se identificó con la intención de cambiar sus hábitos de ingesta para frutas y verduras.

Sólo en el grupo de aquellos estudiantes con estado nutricional normal el género de ellos juega un papel significativo en relación a la conducta que se estaba investigando.

En el grupo con estado nutricional normal se observó que las mujeres se asociaban a las etapas de preparación y acción, respectivamente, mientras que los varones estaban asociados a etapas de precontemplación

\section{TABLA 1}

Características de los estudiantes investigados según género $(\mathbf{n = 1 8 4})$

\begin{tabular}{lcccc} 
CARACTERÍSTICAS & Mujeres $(\mathbf{n = 1 0 4})$ & Hombres $(\mathbf{n = 8 0})$ & Diferencia & $\boldsymbol{P}$ \\
& $\mathrm{X} \pm 1 \mathrm{DE}$ & $\mathrm{X} \pm 1 \mathrm{DE}$ & $\mathbf{\%}$ & $0,1335^{\mathrm{a}}$ \\
\hline Edad (años) & $20,22 \pm 1,86$ & $20,65 \pm 1,98$ & 2,08 & $0,0001^{\text {b }}$ \\
Peso (kilos) & $57,97 \pm 8,34$ & $75,82 \pm 10,45$ & 23,54 & $<0,0001^{\text {a }}$ \\
Talla (metros) & $1,60 \pm 0,06$ & $1,73 \pm 0,06$ & 7,51 & $<0,0001^{\text {a }}$ \\
IMC (kg/mt $\left.{ }^{2}\right)$ & $22,60 \pm 3,05$ & $25,22 \pm 3,22$ & 10,35 &
\end{tabular}

a t-Student; ${ }^{\mathrm{b}}$ Mann-Whitney

\section{TABLA 2}

Etapas del cambio conductual en relación a ingesta de frutas y verduras, control de peso y ejercicio físico según género de los estudiantes de la Universidad del Desarrollo

\begin{tabular}{|c|c|c|c|c|c|c|}
\hline \multirow{2}{*}{$\begin{array}{l}\text { Etapas del } \\
\text { Cambio }\end{array}$} & \multicolumn{2}{|c|}{ Frutas y Verduras } & \multicolumn{2}{|c|}{ Control De Peso } & \multicolumn{2}{|c|}{ Ejercicio Físico } \\
\hline & $\begin{array}{c}\text { Hombres } \\
\mathrm{n}(\%)\end{array}$ & $\begin{array}{c}\text { Mujeres } \\
\mathrm{n}(\%)\end{array}$ & $\begin{array}{l}\text { Hombres } \\
\mathrm{n}(\%)\end{array}$ & $\begin{array}{c}\text { Mujeres } \\
\mathrm{n}(\%)\end{array}$ & $\begin{array}{c}\text { Hombres } \\
\mathrm{n}(\%)\end{array}$ & $\begin{array}{c}\text { Mujeres } \\
\mathrm{n}(\%)\end{array}$ \\
\hline Precontemplación & $34(42,5)$ & $33(31,7)$ & $47\left(58,8^{*}\right)$ & $28(26,9)$ & $3(3,8)$ & $9(8,7)$ \\
\hline Contemplación & $9(11,2)$ & $8(7,7)$ & $15(18,8)$ & $26(25,0)$ & $3(3,8)$ & $20\left(19,2^{* * *}\right)$ \\
\hline Preparación a la Acción & $37(46,3)$ & $62\left(59,6^{*}\right)$ & $10(12,5)$ & $29\left(27,9^{* * *}\right)$ & $9(11,2)$ & $25\left(24,0^{* * *}\right)$ \\
\hline Acción & 0 & $1(0,96)$ & $8(10,0)$ & $21\left(20,2^{* *}\right)$ & $13(16,2)$ & $28\left(26,9^{*}\right)$ \\
\hline Mantenimiento & $0^{£}$ & $0^{£}$ & $0^{£}$ & $0^{f}$ & $52\left(65,0^{* * * *}\right)$ & $22(21,2)$ \\
\hline TOTAL & $80(100)$ & $104(100)$ & $80(100)$ & $104(100)$ & $80(100)$ & 104 (100) \\
\hline $\begin{array}{l}\text { Prueba de } \\
\text { Asociación }\end{array}$ & \multicolumn{2}{|c|}{$\begin{array}{l}\text { V de Cramer:0,153; } \\
\qquad \mathrm{P}=0,228\end{array}$} & \multicolumn{2}{|c|}{$\begin{array}{c}\mathrm{Chi}^{2}=20,059 ; \mathrm{gl}=3 ; \\
P<0,001\end{array}$} & \multicolumn{2}{|c|}{$\begin{array}{c}\mathrm{Chi}^{2}=38,265 ; \mathrm{gl}=4 \\
P<0,001\end{array}$} \\
\hline
\end{tabular}


y contemplación $(\mathrm{p}<0,05)$. En el resto de los grupos de estado nutricionales no existió evidencia suficiente que permitiera afirmar una asociación entre el género de los estudiantes con las etapas del cambio conductual en relación a la ingesta de frutas y verduras $(\mathrm{P}=0,412$ en sobrepeso y $\mathrm{P}=0,394$ para obesos).

En la tabla 4 se observa que el $40,76 \%$ de los estudiantes se encontraban en la etapa de precontemplación en relación a la conducta de control de peso $(n=75)$, mientras el 15,76\% estaba en la etapa de acción $(n=29)$.

El género de los estudiantes con estado nutricional normal presentó una asociación significativa respecto a las etapas del cambio conductual en relación al control de peso $(\mathrm{p}<0,001)$. Es así como las mujeres se concentran preferentemente en las etapas de contemplación, preparación a la acción y acción, sumando un 34,79\% de la muestra. Por otra parte, los varones se les identificaron mayoritariamente con la etapa de precontemplación
$(15,76 \%$ de la muestra; $n=29)$. Todas las estudiantes con bajo peso se las identificó en la etapa de precontemplación respecto al control de peso, lo que constituye un antecedente importante a la hora de manejar la condición dietoterapéutica en particular de ellas.

La tabla 5 muestra que el 40,2 \% de los estudiantes mantiene la acción respecto al ejercicio físico $(n=74)$. Además, se pone en evidencia que en los estudiantes con buen estado nutritivo se registra una asociación significativa del género respecto a las etapas de la conducta referente al ejercicio físico $(\mathrm{p}<0,001)$, donde las mujeres se asocian a las etapas de precontemplación, contemplación, preparación a la acción y acción, sumando un 37,49\% (69 de 184 estudiantes). Por otro lado, el $15,22 \%$ de todos los estudiantes son varones eutróficos que fueron identificados con la etapa de mantenimiento de la acción, conducta que avisora un empoderamiento eficaz de la dimensión del ejercicio físico $(n=28)$.

\section{TABLA 3}

\section{Etapas del cambio conductual en relación a la ingesta de frutas y verduras según género y estado nutricional de los estudiantes de la Universidad del Desarrollo}

\begin{tabular}{|c|c|c|c|c|c|c|c|}
\hline $\begin{array}{l}\text { Estado } \\
\text { nutricional }\end{array}$ & Género & $\begin{array}{c}\text { Precontem } \\
\text { plación } \\
\text { n }(\%)\end{array}$ & $\begin{array}{c}\text { Contem } \\
\text { plación } \\
\text { n }(\%)\end{array}$ & $\begin{array}{c}\text { Preparar } \\
\text { Acción } \\
\text { n }(\%)\end{array}$ & $\begin{array}{l}\text { Acción } \\
\text { n (\%) }\end{array}$ & $\begin{array}{c}\text { Manteni } \\
\text { miento } \\
\mathrm{n}(\%)\end{array}$ & $\begin{array}{l}\text { Total } \\
\text { n }(\%)\end{array}$ \\
\hline \multirow[t]{3}{*}{ Enflaquecido $^{\mathfrak{f}}$} & Femenino & $3(1,63)$ & 0 & $1(0,54)$ & 0 & 0 & $4(2,17)$ \\
\hline & Masculino & 0 & 0 & 0 & 0 & 0 & $0(\mathbf{0 , 0 0})$ \\
\hline & TOTAL & $3(1,63)$ & $\mathbf{0}(\mathbf{0 , 0 0 )}$ & $1(0,54)$ & $0(0,00)$ & $0(0,00)$ & $4(2,17)$ \\
\hline Normal & Femenino & $25(13,59)$ & $5(2,72)$ & $54(29,35)$ & $1(0,54)$ & 0 & $85(67,5)$ \\
\hline \multirow[t]{2}{*}{$\mathrm{V}=\mathbf{0 , 2 6 4} ; P=0,032$} & Masculino & $18(9,78)$ & $7(3,80)$ & $16(8,70)$ & 0 & 0 & $41(32,5)$ \\
\hline & TOTAL & $43(23,37)$ & $12(6,52)$ & $70(38,04)$ & $1(100)$ & $0(0,00)$ & $126(100)$ \\
\hline Sobrepeso & Femenino & $4(2,17)$ & $2(1,09)$ & $4(2,17)$ & 0 & 0 & $10(23,8)$ \\
\hline \multirow[t]{2}{*}{$V=0,206 ; P=0,412$} & Masculino & $13(7,07)$ & $2(1,09)$ & $17(9,24)$ & 0 & 0 & $32(76,2)$ \\
\hline & TOTAL & $17(9,24)$ & $4(2,17)$ & $21(11,41)$ & $0(0,00)$ & $0(\mathbf{0 , 0 0})$ & $42(100)$ \\
\hline Obeso & Femenino & $1(0,54)$ & $1(0,54)$ & $3(1,63)$ & 0 & 0 & $5(41,7)$ \\
\hline \multirow[t]{2}{*}{$\mathrm{V}=0,394 ; \mathrm{P}=0,394$} & Masculino & $3(1,63)$ & 0 & $4(2,17)$ & 0 & 0 & $7(58,3)$ \\
\hline & TOTAL & $4(2,17)$ & $1(0,54)$ & $7(3,80)$ & $\mathbf{0}(\mathbf{0 , 0 0 )}$ & $0(0,00)$ & $12(100)$ \\
\hline TOTAL GENERAL & & $67(36,41)$ & $17(9,24)$ & $99(53,80)$ & $1(0,54)$ & $0(0,00)$ & $184(100)$ \\
\hline
\end{tabular}




\section{DISCUSIÓN}

Dado que el perfil antropométrico de los sujetos estudiados difiere significativamente según género, se decidió incluir esta variable al cruce de estado nutricional con cada una de las tres dimensiones. En cuanto a la etapa conductual en la ingesta de frutas y verduras, Di Noia et al indicaron que el instrumento predice efectivamente la conducta de los sujetos, con evidencia en diversas poblaciones (14). Bajo esta premisa, se denota que el género por sí solo no se asocia de manera significativa respecto a las etapas del cambio conductual respecto a la ingesta de frutas y verduras; sin embargo, son más mujeres $(59,6 \%)$ quienes se encuentran en la etapa de preparación a la acción, a diferencia de los hombres $(46,3 \%, \mathrm{p}<0,05)$. Ellas no han considerado seriamente la idea de cambiar la conducta; piensan que las frutas y verduras no le perjudican y no están preocupadas del tema (10), lo que se explica probablemente por la lejanía de la consecuencia que le traerá si no lo adopta como parte de sus quehaceres diarios, dado que su estado nutricional normal le es garantía de buena salud, por lo que almacena la importancia de consumir frutas y verduras, pero no genera la acción efectiva.

Una vez que se agrega el estado nutricional al análisis, se demuestra que los hombres con estado nutricional normal se asocian significativamente con etapas de pre y contemplación, mientras que las mujeres de la misma categoría se asocian a conductas de preparación a la acción y acción de ésta, lo que indica que esta dimensión no es valorada por los estudiantes como factor protector de enfermedades cardiovasculares y cáncer (22-25).

En general, la población sabe lo que hay que comer para tener una alimentación saludable (así lo demuestran repetidas encuestas) y también conoce los beneficios de la actividad física, pero sigue alimentándose mal (26), tal como se observa en el estudio de Olivares y colaboradores donde indicó que sólo el 5\% de los estudiantes de educación superior consume 5 porciones de frutas y

\section{TABLA 4}

\section{Etapas del cambio conductual en relación al control de peso según género y estado nutricional de los estudiantes de la Universidad del Desarrollo}

\begin{tabular}{|c|c|c|c|c|c|c|c|}
\hline $\begin{array}{l}\text { Estado } \\
\text { nutricional }\end{array}$ & Género & $\begin{array}{c}\text { Precontem } \\
\text { plación } \\
\text { n }(\%)\end{array}$ & $\begin{array}{c}\text { Contem } \\
\text { plación } \\
\text { n }(\%)\end{array}$ & $\begin{array}{c}\text { Preparar } \\
\text { Acción } \\
\text { n (\%) }\end{array}$ & $\begin{array}{l}\text { Acción } \\
\text { n (\%) }\end{array}$ & $\begin{array}{c}\text { Manteni } \\
\text { miento } \\
\mathrm{n}(\%)\end{array}$ & $\begin{array}{l}\text { Total } \\
\text { n }(\%)\end{array}$ \\
\hline \multirow[t]{3}{*}{ Enflaquecido $^{f}$} & Femenino & $4(2,17)$ & 0 & 0 & 0 & 0 & $4(2,17)$ \\
\hline & Masculino & 0 & 0 & 0 & 0 & 0 & $0(\mathbf{0 , 0 0 )}$ \\
\hline & TOTAL & $4(2,17)$ & $\mathbf{0}(\mathbf{0 , 0 0 )}$ & $\mathbf{0}(\mathbf{0 , 0 0 )}$ & $0(0,00)$ & $0(0,00)$ & $4(2,17)$ \\
\hline Normal & Femenino & $21(11,41)$ & $19(10,33)$ & $25(13,59)$ & $20(10,87)$ & 0 & $85(46,20)$ \\
\hline $\mathrm{Chi}^{2}=26,119 ; \mathrm{gl}=3$ & Masculino & $29(15,76)$ & $6(3,26)$ & $2(1,09)$ & $4(2,17)$ & 0 & $41(22,48)$ \\
\hline$P<0,001$ & TOTAL & $50(21,17)$ & $25(13,59)$ & $27(14,67)$ & $24(13,04)$ & $0(\mathbf{0 , 0 0 )}$ & $126(68,48)$ \\
\hline Sobrepeso & Femenino & $3(1,63)$ & $4(2,17)$ & $3(1,63)$ & 0 & 0 & $10(5,43)$ \\
\hline \multirow[t]{2}{*}{$\mathrm{V}=0,236 ; \mathrm{P}=0,503$} & Masculino & $16(8,70)$ & $8(4,35)$ & $6(3,26)$ & $2(1,09)$ & 0 & $32(17,39)$ \\
\hline & TOTAL & $19(10,33)$ & $12(6,52)$ & $9(4,89)$ & $2(1,09)$ & $0(0,00)$ & $42(22,83)$ \\
\hline Obeso & Femenino & 0 & $3(1,63)$ & $1(0,54)$ & $1(0,54)$ & 0 & $5(2,72)$ \\
\hline \multirow[t]{2}{*}{$\mathrm{V}=0,535 ; \mathrm{P}=0,330$} & Masculino & $2(1,09)$ & $1(0,54)$ & $2(1,09)$ & $2(1,09)$ & 0 & $7(3,80)$ \\
\hline & TOTAL & $2(1,09)$ & $4(2,17)$ & $3(1,63)$ & $3(1,63)$ & $0(0,00)$ & $12(6,52)$ \\
\hline TOTAL GENERAL & & $75(40,76)$ & $41(22,28)$ & $39(21,20)$ & $29(15,76)$ & $0(0,00)$ & $184(100)$ \\
\hline
\end{tabular}


verduras al día y al mismo tiempo se les identificó con la conducta de mantenimiento y acción (20). Es por ello la importancia de la campaña "cinco al día", como un intento para promover el consumo de frutas y verduras en grupos específicos de la población que, según la experiencia en Estados Unidos, se observa un efecto promotor en la cantidad de frutas y vegetales consumidas (27). La cantidad de frutas y verduras que consume la población norteamericana está en torno a 3,4 porciones diarias, considerándose bajo la recomendación (28). La experiencia española también indica un aumento del consumo de frutas y verduras, en paralelo a un $55 \%$ de recordación de la campaña promocional cinco al día (29). La información disponible en la Encuesta Nacional de Salud no indaga en la cantidad de porciones diaria de frutas y verduras, razón por la cual es necesario conocer este aspecto para saber si realmente se adecuan a las recomendaciones que promueve la OMS de $400 \mathrm{~g}$ de frutas y verduras al día, que equivalen a dos porciones de frutas y tres de verduras (30). La evidencia existente en el país para la población adulta es escasa y focalizada en muestras específicas, destacando tres estudios que indican una ingesta menor a lo recomendado (25).

En cuanto al control de peso, se observa que también los hombres con estado nutricional normal presentan patrones de conducta ligadas a la precontemplación y contemplación, mientras que las mujeres se asocian a conductas de preparación a la acción y acción, lo que refuerza el tema que el estado nutricional lo perciben como un factor protector y se amparan en su condición para no tomar una actitud de acción ni de mantenimiento de la acción. La contemplación se caracteriza por la intención de realizar la acción, pero por algún motivo no la ejecuta (10).

Al estudiar la dimensión ejercicio físico, se observa que la significación estadística pasa por aquellas personas que presentaron buen estado nutritivo, pero esta vez son los hombres quienes presentaron conductas de

\section{TABLA 5}

\section{Etapas del cambio conductual en relación al ejercicio físico según género y estado nutricional de los estudiantes de la universidad del desarrollo}

\begin{tabular}{|c|c|c|c|c|c|c|c|}
\hline $\begin{array}{l}\text { Estado } \\
\text { nutricional }\end{array}$ & Género & $\begin{array}{c}\text { Precontem } \\
\text { plación } \\
\text { n }(\%)\end{array}$ & $\begin{array}{c}\text { Contem } \\
\text { plación } \\
\text { n (\%) }\end{array}$ & $\begin{array}{c}\text { Preparar } \\
\text { Acción } \\
\text { n }(\%)\end{array}$ & $\begin{array}{l}\text { Acción } \\
\text { n (\%) }\end{array}$ & $\begin{array}{c}\text { Manteni } \\
\text { miento } \\
\mathrm{n}(\%)\end{array}$ & $\begin{array}{l}\text { Total } \\
\text { n (\%) }\end{array}$ \\
\hline \multirow[t]{3}{*}{ Enflaquecido $^{\mathfrak{f}}$} & Femenino & 0 & $1(0,54)$ & 0 & 0 & $3(1,63)$ & $4(2,17)$ \\
\hline & Masculino & 0 & 0 & 0 & 0 & 0 & $0(0,00)$ \\
\hline & TOTAL & $0(0,00)$ & $1(0,54)$ & $0(0,00)$ & $0(0,00)$ & $3(1,63)$ & $4(2,17)$ \\
\hline Normal & Femenino & $7(3,80)$ & $15(8,15)$ & $23(12,5)$ & $24(13,04)$ & $16(8,70)$ & $85(46,5)$ \\
\hline \multirow[t]{2}{*}{$\mathrm{V}=\mathbf{0 , 4 9 4} ; P<0,001$} & Masculino & $1(0,54)$ & $1(0,54)$ & $5(2,72)$ & $6(3,26)$ & $28(15,22)$ & $41(22,28)$ \\
\hline & TOTAL & $8(4,35)$ & $16(8,70)$ & $28(15,22)$ & $30(16,3)$ & $44(23,91)$ & $126(68,48)$ \\
\hline Sobrepeso & Femenino & $1(0,54)$ & $3(1,63)$ & $1(0,54)$ & $3(1,63)$ & $2(1,09)$ & $10(5,43)$ \\
\hline \multirow[t]{2}{*}{$\mathrm{V}=0,438 ; \mathrm{P}=0,089$} & Masculino & $2(1,09)$ & $2(1,09)$ & $3(1,63)$ & $4(2,17)$ & $21(11,41)$ & $32(17,39)$ \\
\hline & TOTAL & $3(1,63)$ & $5(2,72)$ & $4(2,17)$ & $7(3,80)$ & $23(12,5)$ & $42(22,83)$ \\
\hline Obeso & Femenino & $1(0,54)$ & $1(0,54)$ & $1(0,54)$ & $1(0,54)$ & $1(0,54)$ & $5(2,72)$ \\
\hline \multirow[t]{2}{*}{$\mathrm{V}=0,561 ; \mathrm{P}=0,438$} & Masculino & 0 & 0 & $1(0,54)$ & $3(1,63)$ & $3(1,63)$ & $7(3,80)$ \\
\hline & TOTAL & $1(0,54)$ & $1(0,54)$ & $2(1,09)$ & $4(2,17)$ & $4(2,17)$ & $12(6,52)$ \\
\hline TOTAL GENERAL & & $12(6,52)$ & $23(12,50)$ & $34(18,48)$ & $41(22,28)$ & $74(40,22)$ & $184(100)$ \\
\hline
\end{tabular}


mantenimiento de la acción, mientras que las mujeres se asocian a etapas de precontemplación, contemplación, preparación a la acción y acción. La etapa de preparación a la acción, indica que la persona tiene un planteamiento serio de cambio, generalmente asociado a la aparición de síntomas o experiencias cercanas de enfermedad, mientras que en el caso de la etapa de acción, ha realizado los pasos necesarios para cambiar desde hace menos de seis meses (10). Se considera como parte de la explicación a la época donde se realizó el estudio, en que se observa que las mujeres practican mayor actividad física en un tiempo y objetivo específico, que implica un tema estético, lo que indica las conductas de acción a diferencia de los hombres, que conciben al ejercicio físico como parte integral de su conducta, ya que ellos manifiestan realizar actividad física regular en tiempo libre desde hace más de seis meses. (10). Por lo tanto la motivación eficaz por la cual llame al individuo a evolucionar en su conducta lleva a un mantenimiento de la acción, tal como lo señalan Adams y White (31).

En los estudiantes con malnutrición por exceso no se observó diferencias significativas respecto a las etapas de la conducta en las tres dimensiones estudiadas en hombres y mujeres, probablemente debido a que su condición nutricional en unas personas le es interpretada como un freno para llevar a cabo una determinada conducta y en otros se convierte en un factor gatillante. En consecuencia, se hace necesario seguir estudiando otras posibles causas que expliquen tal conducta a este grupo específico de personas.

\section{RESUMEN}

Antecedentes. El estudio de la conducta relacionada con el consumo de frutas y verduras, control de peso y ejercicio físico en adultos jóvenes siguiendo el Modelo Transteórico de Prochaska es un aspecto determinante al momento de evaluar el impacto que estas tres dimensiones presentan como factores preventivos en el combate de enfermedades ligadas al aparato circulatorio y cáncer. Objetivo. Analizar las etapas de cambio conductual referente al consumo de frutas y verduras, control de peso y ejercicio físico en estudiantes universitarios según género y estado nutricional. Diseño. Corte analítico transversal, con un diseño estratificado a 184 estudiantes de ambos sexos entre 18 a 28 años. Resultados. Mujeres con estado nutricional normal se asociaron significativamente con la etapa de "preparación a la acción” $(13,59 \%)$ y “acción” $(10,87 \%)$ respecto al control de peso; los hombres con buen estado nutritivo se asociaron con el "mantenimiento" del ejercicio físico (15,22\% de los 184 estudiantes). El 59,6\% de las mujeres y el 46,3\% de los varones están en preparación para la acción en cuanto al consumo de frutas y verduras $(\mathrm{p}<0,05)$. Conclusión. Hombres y mujeres perciben que sólo un estado nutricional normal es garantía de buena salud; ya que los varones no asignan importancia en el largo plazo al control de peso, ni las mujeres le asignan relevancia en el tiempo al ejercicio físico.

Palabras claves: Modelo Transteórico, frutas y verduras, control de peso, ejercicio físico, género.

Dirigir la correspondencia a:

Profesor

Nut Miguel Ángel López Espinoza

Escuela de Nutrición y Dietética

Facultad de Odontología

Universidad del Desarrollo

Barros Arana $1735,4^{\circ}$ Piso

Concepción, Chile

Fono: (41) 2268583

Fax: (41) 2268501

malopez@udd.cl

Agradecimientos: El autor desea agradecer a la Profesora Sonia Olivares, de la Universidad de Chile por facilitar la encuesta utilizada en este trabajo y a las estudiantes promoción 2007 de la Carrera de Nutrición y Dietética de la Universidad del Desarrollo por el trabajo en terreno.

\section{BIBLIOGRAFÍA}

1. World Health Organization. Dietary changes and exercise needed to counter chronic diseases worldwide. Rev Panam Salud Publ. 2003; 13(5): 346-348. <online> [SCIELO] Consulta: 05/12/2007

2. Cuende Jose I, Acebal Alfredo, Carrera-Camarón Azucena, Salado-Cuadrado Florentino, Díez-Cordero Francisco. et al. La adopción de los percentiles de riesgo cardiovascular evita la sobreestimación o infraestimación del riesgo cardiovascular calculado con el SCORE. Rev Esp Salud Publica 2006; 80(2):125-138.

3. Pilon, André Francisco. The development of health education: an updating of concepts. Rev Saúde Pública 1986; 20(5):391-396.

4. M. Bulló Bonet. La leptina en la regulación del balance energético. Nutr Hosp 2002, 17:42-48. <online> [SCIELO] Consulta: 12/11/2007

5. Atalah, Eduardo. De la desnutrición a la Obesidad: evolución de los problemas nutricionales en Chile. $<$ Presentación $>$ Departamento de Nutrición, Facultad de Medicina. Universidad de Chile. <online> [http://www.minsal.cl/ici/nutricion...] Consulta: $22 / 10 / 2007$ 
6. Fagalde H María del Pilar, Solar H José Antonio del, Guerrero B Marcia, Atalah S Eduardo, et al. Factores de riesgo de enfermedades crónicas no transmisibles en funcionarios de una empresa de servicios financieros de la Región Metropolitana. Rev Méd Chile 2005; 133(8):919-928.

7. Salinas C. Judith, Vio del R. Fernando. Health promotion in Chile. Rev Chil Nut 2002; 29(1): 164-173.

8. Ministerio de Salud, Chile. II Encuesta Nacional de Calidad de Vida y Salud 2006. Departamento de Epidemiología y Departamento de Promoción y Participación Ciudadana.<online>: [http://epi. minsal.cl/epi] Consulta: 24/10/2007

9. Olivares C Sonia, Bustos Nelly Z, Moreno H Ximena, Lera M Lydia, Cortez F Silvana. Actitudes y prácticas sobre alimentación y actividad física en niños obesos y sus madres en Santiago, Chile. Rev Chil Nutr 2006; 33(2):170-179.

10. Cabrera A, Gustavo A. El modelo transteórico del comportamiento en salud. Rev Fac Nac Salud Pública 2000; 18(2): 129-138<online> [http:// guajiros.udea.edu.co/revista/vol_18-2/10182.pdf] Consulta: 21/11/2007

11. Ministerio de Salud. Tabaco o Salud Bucal. Consejería en Tabaquismo. Guía para el Odontólogo. Santiago <online>[http://www.redsalud.gov.cl/ archivos/dental_tabaco.pdf] Consulta: 04/01/2008

12. Hargreaves MK, Schlundt DG, Buchowski MS, Hardy RE, Rossi SR, Rossi JS. Stages of change and the intake of dietary fat in African-American women: improving stage assignment using the Eating Styles Questionnaire. J Am Diet Assoc 1999; 99(11):1392-1399.

13. Finckenor M, Byrd-Bredbenner C. Nutrition intervention group program based on preaction-stageoriented change processes of the Transtheoretical Model promotes long-term reduction in dietary fat intake. J Am Diet Assoc 2000; 100(3):335-342.

14. Jennifer Di Noia, Steven P. Schinke, James O. Prochaska, Isobel R. Contento Application of the Transtheoretical Model to Fruit and Vegetable Consumption Among Economically Disadvantaged African-American Adolescents: Preliminary Findings. Am J Health Promot 2006; 20(5): 342-348.

15. Marcus BH, Simkin LR. The transtheoretical model: applications to exercise behavior. Med Sci Sports Exerc. 1994; 26(11):1400-4.

16. Plotnikoff RC, Hotz SB, Birkett NJ, Courneya KS. Exercise and the transtheoretical model: a longitudinal test of a population sample. Prev Med 2001;
33(5):441-452.

17. Wakui S, Shimomitsu T, Odagiri Y, Inoue S, Takamiya T, Ohya Y. Relation of the stages of change for exercise behaviors, self-efficacy, decisionalbalance, and diet-related psycho-behavioral factors in young Japanese women. J Sports Med Phys Fitness 2002; 42(2):224-32. <online> [PUBMED] Consulta: 13/11/2007

18. Cassidy CA. Facilitating behavior change. Use of the transtheoretical model in the occupational health setting. AAOHN J. 1997; 45(5):239-46.

19. Araya L Héctor, Atalah S Eduardo, Benavides M Xenia, et al. Food and nutrition intervention priorities in Chile. Rev Chil Nutr 2006; 33(3):458-463.

20. Olivares C, Sonia, Lera M, Lydia y Bustos Z, Nelly. Etapas del cambio, beneficios y barreras en actividad física y consumo de frutas y verduras en estudiantes universitarios de Santiago de Chile. Rev Chil Nutr 2008; 35 (1):25-35.

21. Barrera A., M. Gladys. Estándares antropométricos para evaluación del estado nutritivo. Instituto de Nutrición y Tecnología de los Alimentos. Santiago, 2001.

22. Van Duyn MA, Pivonka E. Overview of the health benefits of fruit and vegetable consumption for the dietetics professional: selected literature (Abstract). J Am Diet Assoc 2000;100(12):1511-21 . <online> [PUBMED] Consulta: 15/11/2007

23. Jacoby, Enrique; Keller, Ingrid. La promoción del consumo de frutas y verduras en América Latina: buena oportunidad de acción intersectorial por una alimentación saludable. Rev Chil Nutr 2006;33 (Supl 1): 226-231.

24. Lock, Karen, Pomerleau, Joceline, Knai, Cécile et al. Mejorando la evidencia de intervenciones y programas efectivos diseñados para aumentar el consumo de frutas y verduras a nivel mundial. Rev Chil Nutr 2006;33 (Supl 1):239-251.

25. Olivares C, Sonia; Bustos Z, Nelly. Consumo de verduras y frutas en grupos específicos de consumidores chilenos: elementos a considerar en su promoción. Rev Chil Nutr 2006; 33 (Supl 1):260-264.

26. Vio del R, Fernando, Salinas C, Judith. Promoción de salud y calidad de vida en Chile: una política con nuevos desafíos. Rev Chil Nutr 2006;33 (Supl 1):252-259.

27. Trudeau E, Kristal AR, Li S, Patterson RE. Demographic and psychosocial predictors of fruit and vegetable intakes differ: implications for dietary interventions. J Am Diet Assoc 1998; 98(12):14121417.

28. Subar AF, Heimendinger J, Patterson BH, Krebs- 
Smith SM, Pivonka E, Kessler R. Fruit and vegetable intake in the United States: the baseline survey of the "Five A Day" for Better Health Program. Am J Health Promot. 1995;9(5):352-360.

29. Serrano de N, Fernanda. Estrategia exitosa del programa «5 al día» en España. Rev Chil. Nutr 2006; 33 (Supl 1):272-275.
30. Zacarías, Isabel; keller, Ingrid; Muzzo, Santiago. Programas 5 al día para la promoción del consumo de verduras y frutas. Rev. Chil. Nutr 2006;33(Supl 1): 222-223.

31. J.Adams; M. White. Why don't stage-based activity promotion interventions work? Health Education Res 2005;20(2):237-243. 